\title{
Reduced Presynaptic Dopamine Activity in Adolescent Dorsal Striatum
}

\author{
Marguerite Matthews', Corina Bondi', Gonzalo Torres ${ }^{2}$ and Bita Moghaddam,*, \\ 'Department of Neuroscience, University of Pittsburgh, Pittsburgh, PA, USA; '2 Department of Neurobiology, University of Pittsburgh, Pittsburgh, PA, \\ USA
}

Adolescence coincides with symptomatic onset of several psychiatric illnesses including schizophrenia and addiction. Excess limbic dopamine activity has been implicated in these vulnerabilities. We combined molecular and dynamic indices of dopamine neurotransmission to assess dopamine function in adolescent rats in two functionally distinct striatal subregions: nucleus accumbens (NAc) and dorsal striatum (DS). In adolescents, we find an overall reduction in dopamine availability selective to the DS. Dopamine release in the DS, but not in the NAc, was less responsive to amphetamine in adolescents compared to adults. The dopamine transporter (DAT) inhibitor, nomifensine, similarly inhibited basal and amphetamine-induced dopamine release in either regions of both the age groups, suggesting that the reduced effectiveness of amphetamine is not due to differences in DAT function. Furthermore, DAT and vesicular monoamine transporter-2 expressions were similar in the DS and NAc of adolescent rats. In contrast, expression of tyrosine hydroxylase $(\mathrm{TH})$ was reduced in the DS, but not in the NAc, of adolescents compared to adults. Behaviorally, adolescents were less sensitive to amphetamine but more sensitive to a TH inhibitor. These data indicate that, in contrast to the general notion that dopamine is hyperactive in adolescents, there is diminished presynaptic dopamine activity in adolescents that is selective to the DS and may result from attenuated TH activity. Given recent reports of altered dopamine activity in associative/dorsal striatum of individuals at a clinically high risk of psychosis, our data further support the idea that dorsal, as opposed to ventral, regions of the striatum are a locus of vulnerability for psychosis.

Neuropsychopharmacology (2013) 38, I344-135I; doi:10.1038/npp.2013.32; published online 6 March 2013

Keywords: tyrosine hydroxylase; learning; schizophrenia; addiction; amphetamine; nucleus accumbens

\section{INTRODUCTION}

The brain undergoes major remodeling during adolescence and early adulthood (Rakic et al, 1994; Andersen et al, 2000), which may account for behavioral changes associated with this developmental period. These changes include increased risk-taking and novelty seeking (Adriani et al, 1998). These behaviors, albeit a component of normal maturation processes, can have negative consequences that lead to increased injuries and mortality rates (Lerner and Galambos, 1998; Spear, 2000). Furthermore, it is during this developmental period that the symptomatic onset of many psychiatric illnesses, such as schizophrenia, occurs. The efforts to design safe and mechanistically driven interventions for these illnesses depend on a better functional understanding of neuronal systems that contribute to adolescent vulnerability. The dopamine system has been strongly implicated in adolescent vulnerability to addiction as well as transition to psychosis (Spear, 2000; Wahlstrom

*Correspondence: Dr B Moghaddam, Department of Neuroscience, University of Pittsburgh, A2 10 Langley Hall, Pittsburgh, PA 15260, USA, Tel: +4I2 624 2653, Fax: +412624 9198, E-mail: bita@pitt.edu Received 27 November 2012; revised 15 January 2013; accepted 16 January 20 I3; accepted article preview online 28 January 2013 et al, 2010; O'Donnell, 2011; Sturman and Moghaddam, 2011; Forbes and Dahl, 2012). The dopamine system has a central role in goal-directed and habitual behavior (Kelley, 2004; Wise, 2005), and undergoes significant remodeling during adolescence, including changes in dopamine turnover, synthesis, and reuptake (Teicher et al, 1993; Andersen et al, 1997). Dysfunction of the dopamine system is associated with schizophrenia, mood, and addictive disorders, symptoms of which often manifest during early or late adolescence (Volkmar, 1996; Pine et al, 2002). In particular, recent PET studies have reported abnormal dopamine synthesis capacity before the onset of psychosis in individuals at a clinically high risk of schizophrenia (Howes et al, 2011a; Howes et al, 2011b).

The purpose of the present study was to better understand the dynamics of dopamine neurotransmission in behaving adolescents using a rodent model. We focused on two functionally distinct, dopamine-innervated striatal subregions: the nucleus accumbens (NAc) and the dorsal striatum (DS). These regions are integral to behaviors that are highly relevant to adolescent vulnerability to psychiatric illnesses. The NAc is implicated in motivation and reward processing (Mogenson et al, 1980), and the DS is involved in habit formation and action selection (Yin et al, 2006a; 
Balleine et al, 2009). Recent studies have described agerelated electrophysiological differences in the reactivity of neurons to salient events in these two regions (Sturman and Moghaddam, 2011). We used amphetamine as a tool to activate dopamine release in behaving adolescent and adult rats. Amphetamine disrupts the dopamine transporter (DAT) (Sulzer et al, 1995), thus increasing the dopamine efflux. Amphetamine-like stimulants are commonly prescribed to human youths with ADHD (Kutcher et al, 2004), though little is known about their impact on dopamine neurotransmission at this age. These stimulants produce smaller behavioral activation in adolescent rodents compared to adults (Bolanos et al, 1998; Laviola et al, 1999). Thus, amphetamine may be an important tool for revealing developmental differences in the release properties of dopamine in behaving adolescents. We find that amphetamine-induced dopamine release is attenuated in the DS of adolescents compared to adults. This effect appears to be due to reduced tyrosine hydroxylase $(\mathrm{TH})$ expression in the DS. Collectively, our data point to a DS-selective hypodopaminergic state in adolescents.

\section{MATERIALS AND METHODS}

\section{Subjects}

Adolescent (PND 34-38) and adult (PND 70-80) male Sprague-Dawley rats (Harlan, Frederick, MD) were pairhoused in a $12 \mathrm{~h}$ light/dark cycle (lights on at 1900 hours). Experiments were conducted during the animals' dark (active) phase. All animals were allowed to acclimate to the housing environment for at least 5 days prior to experiments or sacrifice, and had ad libitum access to water and rat chow food. All microdialysis experiments and western blot analyses employed separate subsets of rats, and there was no overlap of subjects within these experiments. Experiments were performed in accordance with and approved by the ethical guidelines of the Institutional Animal Care and Use Committee at the University of Pittsburgh.

\section{Microdialysis Measures of Dopamine in Behaving Rats}

For microdialysis probe implantation, rats were anesthetized with isoflurane and placed in a stereotaxic apparatus using blunt ear bars. All methods and procedures were as described previously (Adams and Moghaddam, 1998). Animals were implanted bilaterally with dialysis probes in both the medial area of the DS (for adolescents: AP +0.7 , ML + 2.0 from bregma, DV -5.0 from skull; and for adults: $\mathrm{AP}+1.6, \mathrm{ML}+2.2$ from bregma, $\mathrm{DV}-6.0$ from skull) and the core of the NAc (for adolescents: AP +1.0 , ML + 1.2 from bregma, and DV - 7.0 from skull; and for adults: $\mathrm{AP}+1.2, \mathrm{ML}+1.1$ from bregma, $\mathrm{DV}-8.4$ from skull). Motor behavior during the microdialysis experiments was quantified as described before (Pehrson and Moghaddam, 2010). Ambulations were defined as movements where the animal moved its entire body (ie, a new beam was broken, whereas a previously broken beam was released). Fine movements were defined as smaller movements where a new beam was broken without releasing a previously broken beam. After completion of microdialysis experiments, rats were anesthetized with chloral hydrate $(400 \mathrm{mg} / \mathrm{kg})$ and perfused intracardially with $0.9 \%$ saline. Stained sections were evaluated for accuracy of probe placement. Only data from placements within the brain region of interest were used for further analysis (Figure 1).

\section{Drugs}

Amphetamine (1.0 mg/kg; Sigma-Aldrich, St Louis, MO), alpha-methyl-DL-tyrosine $(50 \mathrm{mg} / \mathrm{kg}$; TCI America), and nomifensine ( $20 \mu \mathrm{M}$; Sigma-Aldrich) were dissolved in $0.9 \%$ saline. Nomifensine was diluted further $(5 \mu \mathrm{M})$ in Ringer's solution before infusion through the microdialysis probe. The dose of amphetamine used in this study was consistent with previous work, demonstrating that low doses of amphetamine increase the striatal dopamine efflux and associated the motor behaviors (ie, locomotion, stereotyped movements) in adolescent and adult rats. The dose of nomifensine was chosen based on our previous studies that show low doses of nomifensine infusion cause sustained increases in striatal dopamine (Adams et al, 2002). The alpha-methyl-DL-tyrosine dose was chosen based on previous studies, demonstrating that pretreatment with a dose of $50 \mathrm{mg} / \mathrm{kg}$ (i.p.) attenuates the stimulation of locomotor activity induced by amphetamine (Finn et al, 1990; Di Lullo and Martin-Iverson, 1991).

\section{Western Blot Analysis}

Rats were anesthetized with chloral hydrate, brains removed rapidly, and placed in an iced metal brain mold for dissection of NAc and DS. The dissected tissue was homogenized with a Polytron homogenizer in buffer D (20 mM HEPES, $125 \mathrm{mM} \mathrm{NaCl}, 10 \%$ glycerol, $1 \mathrm{mM}$ EDTA, $1 \mathrm{mM}$ EGTA, $1 \mathrm{mM}$ DTT, pH 7.6), containing protease inhibitors (Pierce). Triton X-100 was added to a final concentration of $1 \%$ and the samples were incubated with

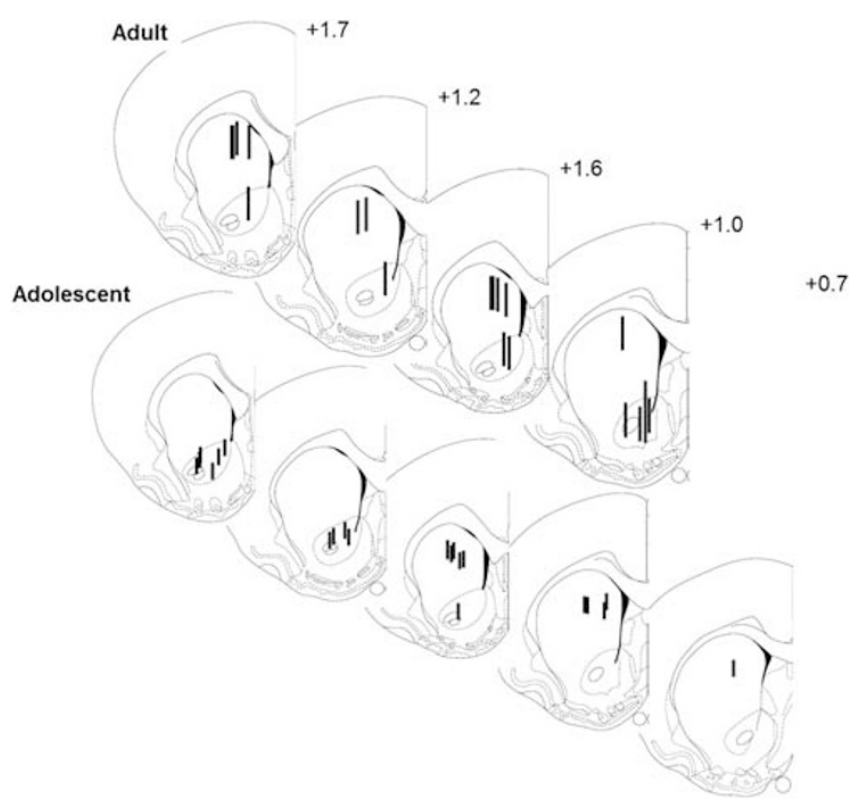

Figure I Histology. Schematic representations of microdialysis probe placements in the DS and NAc. 
rotation for $1 \mathrm{~h}$ at $4{ }^{\circ} \mathrm{C}$. Samples were centrifuged twice at $4{ }^{\circ} \mathrm{C}$ at $10000 \mathrm{~g}$ for $10 \mathrm{~min}$. The supernatant was collected and measured for protein concentration. Samples were separated by SDS-PAGE on $10 \%$ Tris- $\mathrm{HCl}$ polyacrylamide gels and transferred to nitrocellulose membranes using the Bio-Rad system. Nitrocellulose membranes were first blocked for $1 \mathrm{~h}$ in TBS buffer (50 mM Tris- $\mathrm{HCl}, 150 \mathrm{mM}$ $\mathrm{NaCl}, 0.2 \%$ Tween 20 ) containing $5 \%$ dry milk and then incubated with the indicated primary antibody in blocking buffer, washed three times, and incubated with a horseradish peroxidase-conjugated secondary antibody. Following all antibody incubations, membranes were washed three times with TBS buffer and protein bands were visualized using the West Pico SuperSignal system (Pierce). Primary antibodies included anti-TH, anti-VMAT2, anti-DAT, and anti-tubulin.

\section{Data Analysis}

Microdialysis and behavior data were analyzed as described previously (Adams and Moghaddam, 1998; Pehrson and Moghaddam, 2010). The microdialysis data were expressed as a percentage of baseline DA release, where baseline was defined as mean of the three baseline values obtained immediately before a pharmacological manipulation. For amphetamine studies, locomotor activity and stereotypy data were expressed as the number of infrared beam breaks within a 20 -min bin (to correspond with the 20-min microdialysis sample-collection bins). Western blot data were expressed as the optical density ratio of protein normalized to tubulin. Statistical analysis of these dependent measures was conducted using the two-factor repeated measures, ANOVAs with age as the between-subjects factor, and time as the within-subjects factor. For the western blot analysis, independent $t$-tests were used.

\section{RESULTS}

\section{Effects of Amphetamine on Motor Behavior and Dopamine Efflux in the DS and NAc}

Microdialysate analyses of baseline dopamine levels indicated no age-dependent differences in NAc or DS. The baseline means (in $\mathrm{fmol} / \mu \mathrm{l}$ ) for the NAc were $0.48 \pm$ 0.10 in adolescents and $0.60 \pm 0.14$ in adults $\left(t_{19}=-0.693\right.$, n.s.); the baseline mean (in fmol/ $\mu \mathrm{l}$ ) for the DS were $1.13 \pm 0.33$ in adolescents and $0.95 \pm 0.29$ in adults $\left(t_{21}=0.687\right.$, n.s. $)$.

Systemic administration of amphetamine ( $1.0 \mathrm{mg} / \mathrm{kg}$, i.p.) produced different regional effects in dopamine efflux in adolescent $v s$ adult rats. Amphetamine had similar effects on both the age groups in NAc (Figure 2a), whereas it induces significantly lower enhancements in dopamine levels in the DS of adolescent $v s$ adult rats (Figure $2 \mathrm{~b}$; time $\times$ age interaction $F(11,99)=27.30, p<0.001)$. After vehicle injection, there was no significant effect on dopamine efflux in either NAc (Figure 2a) or DS (Figure 2b) of either age group. There were also no age-related differences in resting baseline extracellular levels of dopamine in DS or NAc.

In the same animals, amphetamine produced smaller activation of locomotion (Figure $2 \mathrm{c}$; time $\times$ age interaction
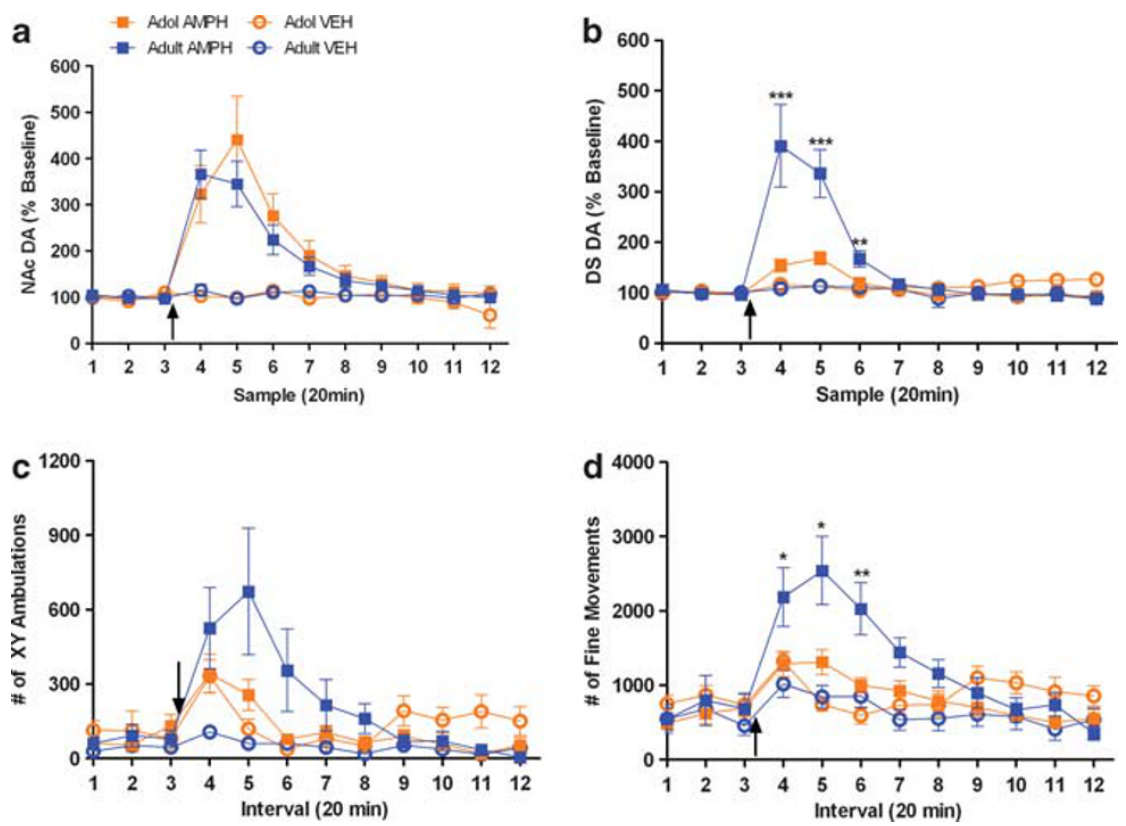

Figure 2 Amphetamine causes lower increases in DS dopamine and motor behavior in adolescent rats. Adolescent (orange) and adult (blue) rats were given an i.p. injection of $1.0 \mathrm{mg} / \mathrm{kg}$ amphetamine (squares) or $0.9 \%$ saline vehicle (circles). Dopamine release was measured in the (a) NAc (adolescent amphetamine group, $n=6$; adult amphetamine group, $n=6$; adolescent vehicle group, $n=5$; adult vehicle group, $n=5$ ), and (b) DS (adolescent amphetamine group, $n=6$; adult amphetamine group, $n=5$; adolescent vehicle group, $n=6$; adult vehicle group, $n=5$ ). Motor behavior was also measured for each animal, expressed as (c) ambulations (adolescent amphetamine group, $n=8$; adult amphetamine group, $n=6$; adolescent vehicle group, $n=9$; adult vehicle group, $n=9$ ), and (d) fine movements (adolescent amphetamine group, $n=8$; adult amphetamine group, $n=6$; adolescent vehicle group, $n=9$; adult vehicle group, $n=9$ ). In each graph, baseline dopamine release and motor behavior were measured prior to injection, represented by the first three time points displayed. All data are expressed as mean \pm SEM. Black arrows denote time of injection. Asterisk(s) denotes significant difference between amphetamine-treated age groups at a given time point ( ${ }^{*} p<0.05$, $* * * 0.0$ I, $* * * p<0.00$ I). 
$F(11,132)=2.48, \quad p<0.01)$ and fine motor movements (Figure 2d; time $\times$ age interaction $F(11,132)=3.51$, $p<0.001)$ in adolescents compared to adults. Vehicle administration showed no effect on locomotion (Figure 2c) or fine movements between the groups (Figure 2d), though adolescent rats had a transient response in fine movements immediately after the injection.

\section{Protein Expression Levels of DAT, VMAT2, and TH in the DS and NAc}

Given the region-specific differences in amphetamineinduced dopamine efflux between adolescent and adult rats, we sought to investigate the molecular mechanisms responsible for these differences. We used western blot analysis to measure the levels of proteins involved in dopamine availability and effects of amphetamine, including DAT, the monamine vesicular transporter-2 (VMAT2), and $\mathrm{TH}$ of adolescent and adult rats (Figure 3). Protein analyses showed that adolescents have reduced DAT protein levels in both the NAc (Figure $3 \mathrm{a} ; t_{5}=3.70, p<0.05$ ) and DS (Figure $3 \mathrm{~b} ; t_{6}=4.05, p<0.01$ ) compared to adults. In contrast, TH levels were selectively lower in DS of adolescents compared to adults (Figure $3 \mathrm{~b} ; t_{10}=4.87$, $p<0.001$ ), whereas levels were comparable in NAc of both the age groups (Figure $3 \mathrm{a}$ ). There were no significant differences between either age group in VMAT2 levels in the NAc (Figure $3 a$ ) or in the DS (Figure 3b).

\section{Effects of Nomifensine and Amphetamine on Dopamine Efflux in the DS and NAc}

Although reduced DAT levels were observed in adolescents compared to adults in both the NAc and DS, the difference in the DS was comparably more than the one in NAc (age $\times$ region interaction $F(1,10)=6.95, p<0.05)$, suggesting that the reduced DAT function may potentially account for reduced effects of amphetamine on dopamine release in DS. To test the validity of this mechanism, we compared the effect of locally applied DAT inhibitor, nomifensine, on basal and amphetamine-activated extracellular dopamine levels at doses that produce a stable increase in extracellular levels of dopamine (Adams et al, 2002). In both NAc and DS of adolescents (Figure $4 \mathrm{a}$ and $\mathrm{b}$ ), application of the DAT inhibitor, nomifensine, produced a near six-fold increase in dopamine efflux that was sustained throughout the drug infusion. There were no significant age differences in dopamine efflux in either the NAc (Figure 4a) or DS (Figure 4b), in that a similar magnitude of increase was observed in both adolescents and adults. Amphetamine administration after nomifensine infusion had no agedependent differences in dopamine efflux in NAc (Figure 4c) or DS (Figure 4d). The fact that the local blockade of DAT with nomifensine had similar effects on dopamine release in the NAc and DS between adolescents and adults suggests that the function or density of DAT alone is not responsible for the region-specific effects of amphetamine-induced dopamine release.

\section{Effects of TH Inhibition on Amphetamine-induced Motor Behavior}

To examine the role of $\mathrm{TH}$ regulation on dopaminemediated motor behaviors, animals were pretreated with the TH inhibitor, alpha-methyl-DL-tyrosine, (50 mg/kg, i.p.) for $2 \mathrm{~h}$ prior to amphetamine $(1.0 \mathrm{mg} / \mathrm{kg}$, i.p.). Amphetamine produces hyperactivity primarily by releasing newly synthesized dopamine, and the dose of alpha-methyl-DLtyrosine chosen attenuates the motor-activating effects of amphetamine (Finn et al, 1990; Di Lullo and MartinIverson, 1991). Adults and adolescents pretreated with alpha-methyl-DL-tyrosine responded similarly to amphetamine in locomotor ambulations (Figure 5a), whereas amphetamine-induced stereotyped movements were significantly reduced in adolescents (Figure 5b; time $\times$ age interaction $F(29,206)=1.740, p<0.01)$. Vehicle administration showed no effect on locomotion (Figure 5a) or fine movements between the groups (Figure $5 \mathrm{~b}$ ).
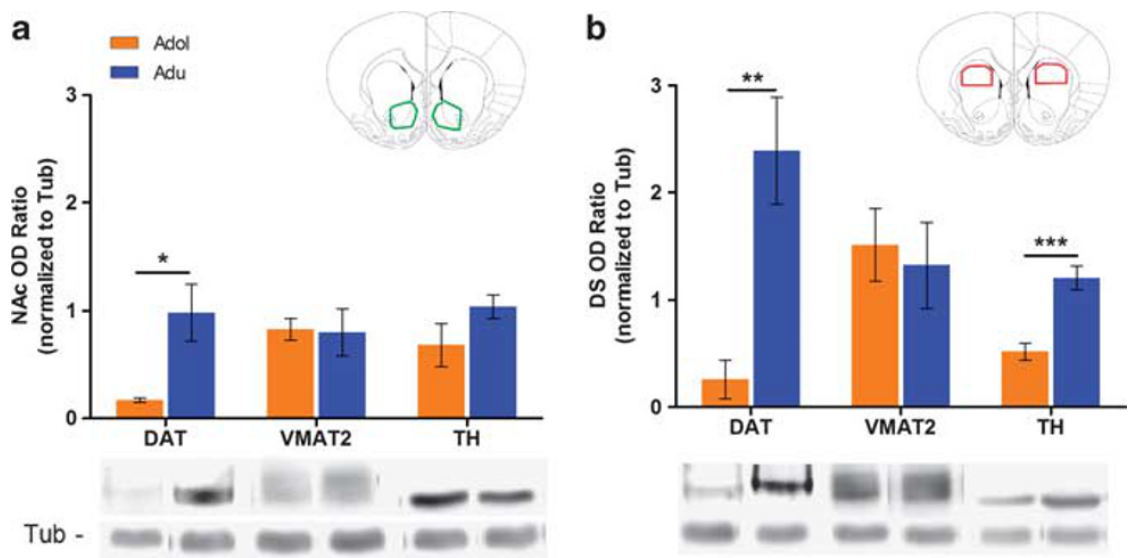

Figure 3 Adolescent rats have decreased levels of TH and DAT in the DS. Diagrams in the upper right corner of each panel depict general regions of dissection of tissue-free hand dissected bilaterally from both the NAc (outlined in green) and DS (outlined in red) of adolescent and adult rats. Protein expression levels of DAT (adolescents, $n=4$; adults, $n=3$ ), VMAT2 ( $n=6$ for each group), and TH ( $n=6$ for each group) were measured using western blot analysis in the (a) NAc, and (b) DS of adolescent (orange bars) and adult (blue bars) rats. All protein optical densities were normalized to tubulin, shown along the bottom of the figure. All data are expressed as mean \pm SEM. Asterisk(s) denotes significant differences between age groups $(* p<0.05$, $* * * 0.0$ I, ****** $p<0.001)$. 

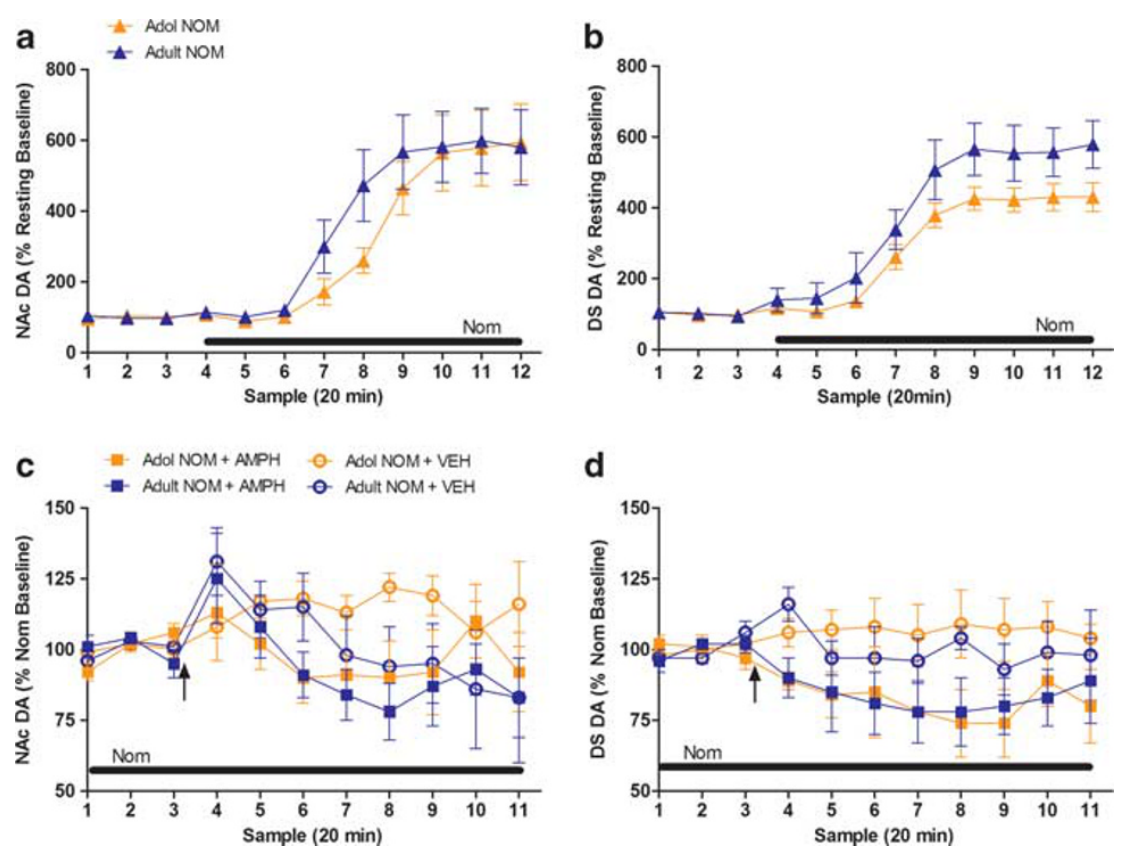

Figure 4 Blockade of DAT produced similar regional effects on dopamine release in both adolescents and adults. The DAT inhibitor nomifensine was infused directly into the NAc and DS of adolescent (orange) and adult (blue) rats. Dopamine release was measured in the (a) NAc (adolescent group, $n=11$; adult group, $n=10$ ), and (b) DS (adolescent group, $n=14$; adult group, $n=11$ ). When maximum dopamine release was reached for at least three consistent time points during nomifensine infusion, animals were given an i.p. injection of $1.0 \mathrm{mg} / \mathrm{kg}$ amphetamine (squares) or $0.9 \%$ saline vehicle (circles). Dopamine release continued to be measured in the (c) NAc (adolescent amphetamine group, $n=6$; adult amphetamine group, $n=6$; adolescent vehicle group, $n=5$; adult vehicle group, $n=5$ ), and (d) DS (adolescent amphetamine group, $n=6$; adult amphetamine group, $n=5$; adolescent vehicle group, $n=8$; adult vehicle group, $n=6$ ) following injection. In graphs (a) and (b), baseline dopamine release was measured prior to infusion, represented by the first three time points displayed. In graphs (c) and (d), baseline dopamine release was measured after nomifensine infusion caused a plateau in dopamine release, represented by the first three time points displayed. All data are expressed as mean \pm SEM. Black bar denotes nomifensine infusion. Black arrow denotes time of amphetamine injection.
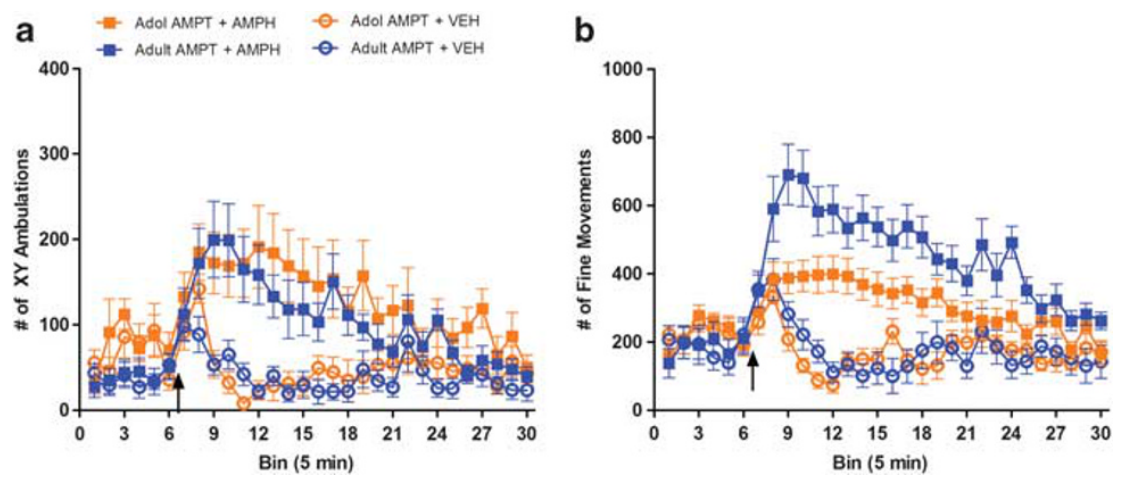

Figure 5 TH inhibition causes lower increases in fine movements in adolescent rats after amphetamine administration. Adolescent (orange) and adult (blue) rats were pretreated with alpha-methyl-DL-tyrosine before given an i.p. injection of $1.0 \mathrm{mg} / \mathrm{kg}$ amphetamine (squares) or $0.9 \%$ saline vehicle (circles). Motor behavior was measured for each animal ( $n=10$ for each group), expressed as (a) ambulation, and (b) fine movements. In each graph, baseline motor behavior during pretreatment was measured prior to amphetamine injection, represented by the first six time points displayed. All data are expressed as mean \pm SEM. Black arrow denotes time of injection.

\section{DISCUSSION}

We find that compared to adults, amphetamine releases less dopamine in the DS of adolescents. Our data further indicate that a potential mechanism for this attenuated response is the reduced expression of TH in the DS of adolescents, which would diminish dopamine synthesis and the releasable pool of dopamine. In contrast to the DS, we find similar levels of $\mathrm{TH}$ expression and amphetamineinduced dopamine release in the NAc of adolescents and adults. Dopamine influences striatal neuronal activity primarily by modulating the impact of cortical and other inputs onto these neurons (Surmeier et al, 2011). Our findings, therefore, suggest that the modulatory role of dopamine is reduced in the DS of adolescents. These data are in contrast to theories that suggest dopamine neurotransmission is enhanced during adolescence (Chambers et al, 2003; Wahlstrom et al, 2010; Forbes and Dahl, 2012). The data, however, are consistent with exaggerated responsiveness of adolescent DS neurons to salient events such as 
reward expectancy (Sturman and Moghaddam, 2012). Reduced dopamine modulation in the DS during this developmental phase may be a 'normal' process that enhances DS-dependent functions, such as actionoutcome learning. Absence of this reduced modulation may serve as a mechanism for increased vulnerability to dopamine-related illnesses like schizophrenia and addiction. Consistent with this hypothesis, recent studies have linked elevated presynaptic measures of dopamine in the DS to prodromal signs of schizophrenia and transition to psychosis in at-risk individuals (Howes et al, 2009; Howes et al, 2011a; Howes et al, 2011b; Allen et al, 2012).

\section{Adolescent Responses to Psychostimulants}

Our finding of age-dependent differences in sensitivity to amphetamine is consistent with studies showing that adolescent rats are less sensitive than adults to the motoractivating effects of psychostimulants (Bolanos et al, 1998; Laviola et al, 1999). The unconditioned behavioral responses to psychostimulants depend on dopamine function (Beninger, 1983). Decreasing or depleting dopamine by blocking the dopamine receptors or lesioning dopamine cells with 6-hydroxy-dopamine produces hypolocomotion, whereas drugs that enhance dopamine neurotransmission, such as amphetamine and cocaine, produce hyperlocomotion and stereotypy (Beninger, 1983). There is a dorsalventral relationship in the striatum related to this effect in that stereotypy and fine movements are attributed to DS dopamine activation, whereas hyperlocomotion is attributed to NAc dopamine activation (Beninger, 1983). Despite the observation that both amphetamine-induced hyperlocomotion and stereotypy were reduced in adolescents, we found a reduction in dopamine release that was selective to the DS. A lack of difference in dopamine release in the NAc suggests that dopamine-mediated behaviors such as locomotion that are dependent on the functional integrity of this region in adults may be differently regulated in adolescents. Our data further indicate indicate that at least some of the observed reduced behavioral effects of stimulants in adolescents may be attributed to hypoactive dopamine neurotransmission in the DS.

\section{Dopamine Maturation Occurs During Adolescence}

The age-specific behavioral differences observed in response to dopamine releasers, such as amphetamine, may be the result of underlying developmental neural changes in the dopamine circuitry during adolescence. Several lines of evidence indicate that the dopamine system undergoes major remodeling during adolescence. In adolescent rodents, there is dopamine receptor overproduction and pruning in the striatal regions (Andersen et al, 1997; Badanich et al, 2006; Cao et al, 2007). The modulatory impact of dopamine receptor-binding shifts from being mildly inhibitory to strongly excitatory during adolescence to early adulthood (O'Donnell, 2011). Firing rates of midbrain dopamine neurons also have been shown to peak during adolescence before decreasing over time into adulthood (McCutcheon and Marinelli, 2009; McCutcheon et al, 2012). Furthermore, DAT levels in both the DS and NAc are considered to follow a similar pattern of postnatal development, with higher DAT levels in adulthood compared to adolescence in these regions (Tarazi et al, 1998). However, there have been other reports that show DAT levels peak in adolescence and gradually decline with age (Meng et al, 1999; Moll et al, 2000).

When considering the action of amphetamine in the present context, it is important to underscore the potential neurodevelopmental consequences of DAT on dopamine availability. Amphetamine increases extracellular dopamine by disrupting the function of DAT (Sulzer et al, 1995). Our finding of reduced amphetamine effect on dopamine efflux in adolescent DS could indicate the reduced DAT function in this region. When examining DAT expression and function, however, we found that adolescents express less DAT in both the NAc and DS than adults, and that the functional consequences of DAT inhibition are similar in both regions. Consistent with these data, Volz et al (2009) have shown that whereas adolescent rats have lower DAT expression in striatal tissue, there are increased levels of functionally active DAT as measured by dopamine transport velocities and DAT immunoreactivity. This study did not distinguish between dorsal and ventral striatum but the results suggest that although adolescent rats express less DAT, these DATs are functionally more efficacious at dopamine clearance than those expressed in adults.

Similar patterns of reuptake in both the NAc and DS suggest that there are other mechanisms that govern dopamine availability in adolescents that selectively target the DS, making it hyporesponsive to stimulants such as amphetamine. Our data strongly indicate that this regionally selective mechanism involves expression of $\mathrm{TH}$. Dopamine synthesis is mediated by $\mathrm{TH}$, a rate-limiting enzyme that converts tyrosine to L-DOPA (Molinoff and Axelrod, 1971). TH activity is sustained until catecholamine neurotransmitters are no longer needed, and thus dopamine synthesis and availability are activity-dependent. We observed that, unlike DAT, there are region-specific differences in TH expression between adolescents and adults. Lower levels of TH in the adolescent DS would lead to reduced dopamine synthesis, potentially leading to reduced dopamine transmission in this region. Our finding that amphetamine-induced stereotypy, a motor behavior dependent on dopamine neurotransmission in the DS (Beninger, 1983), was selectively attenuated in adolescents by a TH inhibitor further supports the lower functional state of $\mathrm{TH}$ activity in this region. $\mathrm{TH}$ is regulated long-term through gene expression and short-term through feedback inhibition by catecholamines, allosteric regulation, and phosphorylation (Kumer and Vrana, 1996). Further studies are needed to establish which of these mechanisms contribute to reduced TH activity in the adolescent DS.

\section{Functional and Clinical Relevance}

The most critical finding of this study is that dopamine neurotransmission may be selectively reduced in the DS but not in the NAc of adolescents. These striatal regions are functionally and structurally distinct. The DS is involved in voluntary motor control, instrumental learning, and habit formation, whereas the NAc is involved in reward and reinforced behaviors (Mogenson et al, 1980; Kelley et al, 2004). At the structural level, the pattern of cortical inputs 
to these regions is distinct. Cortical glutamatergic afferents innervate the striatum in a topographic gradient, where the dorsolateral striatum predominantly receives inputs from sensory and motor cortical areas, and the ventromedial striatum (including NAc) receives inputs from the amygdala and hippocampus (Groenewegen, 1994; Voorn et al, 2004). The different cortical and midbrain influence over the DS compared to the NAc may contribute to dopamine synthesis and, subsequently, activity-dependent release throughout adolescence.

The developmental remodeling of the striatum is of particular interest because of its role in goal-directed behavior. Previous models had implicated the limbic system, in particular the amygdala and the NAc, in functional immaturities of the adolescent brain (Spear, 2000; Chambers et al, 2003). However, the present data, as well as a recent electrophysiology study (Sturman and Moghaddam, 2012), do not find functional differences in the NAc of adolescents as compared to adults. Instead, we find reduced dopamine neurotransmission in the DS, a region that is involved critically in learning, habit formation, and action selections (Yin et al, 2006b). Dopamine's action in the striatum is primarily inhibitory (Bamford et al, 2004). Thus, reduced dopamine activity in this region during adolescence may enhance the function of afferent input that mediates learning and habit formation. Whereas this may be a critical process for normal development, disruption of this reduced dopamine activity may produce vulnerability to illnesses such as schizophrenia. Consistent with this mechanism, recent PET studies have reported selective disruption of dopamine synthesis capacity in associative subregions of the striatum in individuals at high risk to develop schizophrenia (Howes et al, 2009; Fusar-Poli et al, 2010) who transition to psychosis (Howes et al, 2011a; Howes et al, 2011b; Allen et al, 2012), suggesting that dysregulation of dopamine synthesis in the DS, potentially through mechanisms that regulate TH activity, could lead to psychosis in these individuals.

\section{Conclusion}

These data demonstrate that dopamine neurotransmission in the DS of adolescent rodents is hypofunctional compared to adults. These findings support the idea that developmental changes of the dopamine system may contribute to illnesses such as schizophrenia and suggest that a locus of this dopaminergic involvement is the DS. As part of normal development, the reduced inhibitory modulation of DS neurons by dopamine may facilitate DS-dependent functions such as action-outcome learning, but an abnormally hyperactive dopamine modulation in the DS may contribute to increased vulnerability to habit formation, impulsive decision making, and development of psychosis in individuals at high risk for schizophrenia.

\section{ACKNOWLEDGEMENTS}

This research was supported by grant from the US National Institute of Health (R37 MH48404).

\section{DISCLOSURE}

The authors declare no conflict of interest.

\section{REFERENCES}

Adams B, Moghaddam B (1998). Corticolimbic dopamine neurotransmission is temporally dissociated from the cognitive and locomotor effects of phencyclidine. J Neurosci 18: 5545-5554.

Adams BW, Bradberry CW, Moghaddam B (2002). NMDA antagonist effects on striatal dopamine release: microdialysis studies in awake monkeys. Synapse 43: 12-18.

Adriani W, Chiarotti F, Laviola G (1998). Elevated novelty seeking and peculiar d-amphetamine sensitization in periadolescent mice compared with adult mice. Behav Neurosci 112: 1152-1166.

Allen P, Luigjes J, Howes OD, Egerton A, Hirao K, Valli I et al (2012). Transition to psychosis associated with prefrontal and subcortical dysfunction in ultra high-risk individuals. Schizophr Bull 38: 1268-1276.

Andersen SL, Dumont NL, Teicher MH (1997). Developmental differences in dopamine synthesis inhibition by $(+/-)-7-\mathrm{OH}-$ DPAT. Naunyn Schmiedebergs Arch Pharmacol 356: 173-181.

Andersen SL, Thompson AT, Rutstein M, Hostetter JC, Teicher MH (2000). Dopamine receptor pruning in prefrontal cortex during the periadolescent period in rats. Synapse 37: 167-169.

Badanich KA, Adler KJ, Kirstein CL (2006). Adolescents differ from adults in cocaine conditioned place preference and cocaine-induced dopamine in the nucleus accumbens septi. Eur J Pharmacol 550: 95-106.

Balleine BW, Liljeholm M, Ostlund SB (2009). The integrative function of the basal ganglia in instrumental conditioning. Behav Brain Res 199: 43-52.

Bamford NS, Robinson S, Palmiter RD, Joyce JA, Moore C, Meshul CK (2004). Dopamine modulates release from corticostriatal terminals. J Neurosci 24: 9541-9552.

Beninger RJ (1983). The role of dopamine in locomotor activity and learning. Brain Res Rev 6: 173-196.

Bolanos CA, Glatt SJ, Jackson D (1998). Subsensitivity to dopaminergic drugs in periadolescent rats: a behavioral and neurochemical analysis. Brain Res DevBrain Res 111: 25-33.

Cao J, Lotfipour S, Loughlin SE, Leslie FM (2007). Adolescent maturation of cocaine-sensitive neural mechanisms. Neuropsychopharmacology 32: 2279-2289.

Chambers RA, Taylor JR, Potenza MN (2003). Developmental neurocircuitry of motivation in adolescence: a critical period of addiction vulnerability. Am J Psychiatry 160: 1041-1052.

Di Lullo SL, Martin-Iverson MT (1991). Presynaptic dopaminergic neurotransmission mediates amphetamine-induced unconditioned but not amphetamine-conditioned locomotion and defecation in the rat. Brain Res 568: 45-54.

Finn IB, Iuvone PM, Holtzman SG (1990). Depletion of catecholamines in the brain of rats differentially affects stimulation of locomotor activity by caffeine, D-amphetamine, and methylphenidate. Neuropharmacology 29: 625-631.

Forbes EE, Dahl RE (2012). Research Review: altered reward function in adolescent depression: what, when and how? J Child Psychol Psychiatry 53: 3-15.

Fusar-Poli P, Howes OD, Allen P, Broome M, Valli I, Asselin MC et al (2010). Abnormal frontostriatal interactions in people with prodromal signs of psychosis: a multimodal imaging study. Arch Gen Psychiatry 67: 683-691.

Groenewegen HJ, Berendse HW (1994). Anatomical relationships between the prefrontal cortex and the basal ganglia in the rat. In: Thierry A-MGlowinski JGoldman-Rakic PSChristen Y (eds) Motor and Cognitive Functions of the Prefrontal Cortex. Springerverlag: Berlin, Heidelberg, 51-77. 
Howes O, Bose S, Turkheimer F, Valli I, Egerton A, Stahl D et al (2011a). Progressive increase in striatal dopamine synthesis capacity as patients develop psychosis: a PET study. Mol Psychiatry 16: 885-886.

Howes OD, Bose SK, Turkheimer F, Valli I, Egerton A, Valmaggia LR et al (2011b). Dopamine synthesis capacity before onset of psychosis: a prospective [18F]-DOPA PET imaging study. Am J Psychiatry 168: 1311-1317.

Howes OD, Montgomery AJ, Asselin MC, Murray RM, Valli I, Tabraham $\mathrm{P}$ et al (2009). Elevated striatal dopamine function linked to prodromal signs of schizophrenia. Arch Gen Psychiatry 66: 13-20.

Kelley A (2004). Memory and addiction: shared neural circuitry and molecular mechanisms. Neuron 44: 161-179.

Kelley AE, Schochet T, Landry CF (2004). Risk taking and novelty seeking in adolescence: introduction to part I. Ann N Y Acad Sci 1021: 27-32.

Kumer SC, Vrana KE (1996). Intricate regulation of tyrosine hydroxylase activity and gene expression. J Neurochem 67: 443-462.

Kutcher S, Aman M, Brooks SJ, Buitelaar J, van Daalen E, Fegert J et al (2004). International consensus statement on attentiondeficit/hyperactivity disorder (ADHD) and disruptive behaviour disorders (DBDs): clinical implications and treatment practice suggestions. Eur Neuropsychopharmacol 14: 11-28.

Laviola G, Adriani W, Terranova ML, Gerra G (1999). Psychobiological risk factors for vulnerability to psychostimulants in human adolescents and animal models. Neurosci Biobehav Rev 23: 993-1010.

Lerner RM, Galambos NL (1998). Adolescent development: challenges and opportunities for research, programs, and policies. Ann Rev Psychol 49: 413-446.

McCutcheon JE, Conrad KL, Carr SB, Ford KA, McGehee DS, Marinelli M (2012). Dopamine neurons in the ventral tegmental area fire faster in adolescent rats than in adults. J Neurophysiol 108: $1620-1630$.

McCutcheon JE, Marinelli M (2009). Age matters. Eur J Neurosci 29: 997-1014.

Meng SZ, Ozawa Y, Itoh M, Takashima S (1999). Developmental and age-related changes of dopamine transporter, and dopamine D1 and D2 receptors in human basal ganglia. Brain Res 843: 136-144.

Mogenson G, Jones D, Yim C (1980). From motivation to action: functional interface between the limbic system and the motor system. Prog Neurobiol 14: 69-97.

Molinoff PB, Axelrod J (1971). Biochemistry of catecholamines. Ann Rev Biochem 40: 465-500.

Moll GH, Mehnert C, Wicker M, Bock N, Rothenberger A, Ruther E et al (2000). Age-associated changes in the densities of presynaptic monoamine transporters in different regions of the rat brain from early juvenile life to late adulthood. Brain Res Dev Brain Res 119: 251-257.
O'Donnell P (2011). Adolescent onset of cortical disinhibition in schizophrenia: insights from animal models. Schizophr Bull 37: 484-492.

Pehrson AL, Moghaddam B (2010). Impact of metabotropic glutamate $2 / 3$ receptor stimulation on activated dopamine release and locomotion. Psychopharmacology (Berl) 211: 443-455.

Pine DS, Cohen P, Johnson JG, Brook JS (2002). Adolescent life events as predictors of adult depression. J Affect Disord 68: 49-57.

Rakic P, Bourgeois JP, Goldman-Rakic PS (1994). Synaptic development of the cerebral cortex: implications for learning, memory, and mental illness. Prog Brain Res 102: 227-243.

Spear LP (2000). The adolescent brain and age-related behavioral manifestations. Neurosci Biobehav Rev 24: 417-463.

Sturman DA, Moghaddam B (2011). The neurobiology of adolescence: changes in brain architecture, functional dynamics, and behavioral tendencies. Neurosci Biobehav Rev 35: 1704-1712.

Sturman DA, Moghaddam B (2012). Striatum processes reward differently in adolescents versus adults. Proc Natl Acad Sci USA 109: 1719-1724.

Sulzer D, Chen TK, Lau YY, Kristensen H, Rayport S, Ewing A (1995). Amphetamine redistributes dopamine from synaptic vesicles to the cytosol and promotes reverse transport. J Neurosci 15: 4102-4108.

Surmeier DJ, Carrillo-Reid L, Bargas J (2011). Dopaminergic modulation of striatal neurons, circuits, and assemblies. Neuroscience 198: 3-18.

Tarazi FI, Tomasini EC, Baldessarini RJ (1998). Postnatal development of dopamine and serotonin transporters in rat caudateputamen and nucleus accumbens septi. Neurosci Lett 254: 21-24.

Teicher MH, Barber NI, Gelbard HA, Gallitano AL, Campbell A, Marsh E et al (1993). Developmental differences in acute nigrostriatal and mesocorticolimbic system response to haloperidol. Neuropsychopharmacology 9: 147-156.

Volkmar FR (1996). Childhood and adolescent psychosis: a review of the past 10 years. J Am Acad Child Adolesc Psychiatry 35: 843-851.

Volz TJ, Farnsworth SJ, Rowley SD, Hanson GR, Fleckenstein AE (2009). Age-dependent differences in dopamine transporter and vesicular monoamine transporter-2 function and their implications for methamphetamine neurotoxicity. Synapse 63: 147-151.

Voorn P, Vanderschuren LJ, Groenewegen HJ, Robbins TW, Pennartz CM (2004). Putting a spin on the dorsal-ventral divide of the striatum. Trends Neurosci 27: 468-474.

Wahlstrom D, White T, Luciana M (2010). Neurobehavioral evidence for changes in dopamine system activity during adolescence. Neurosci Biobehav Rev 34: 631-648.

Wise RA (2005). Forebrain substrates of reward and motivation. J Comp Neurol 493: 115-121.

Yin H, Knowlton B, BW B (2006a). Inactivation of dorsolateral striatum enhances sensitivity to changes in the action-outcome contingency in instrumental conditioning. Behav Brain Res 166: 189-196. 